\title{
A FREE RANK 4 HOPF ALGEBRA WITH ANTIPODE OF ORDER 4
}

\author{
DAVID E. RADFORD
}

\begin{abstract}
Let $k$ be an arbitrary commutative ring. In this paper we construct a free rank 4 Hopf algebra $H$ over $k$ with antipode of order 4 and also discuss its symmetry. $H$ is not semisimple.
\end{abstract}

The purpose of this paper is to describe a free rank 4 Hopf algebra over an arbitrary commutative ring $k$ with antipode of order 4 . Examples of infinite dimensional Hopf algebras over a field $k$ with antipode of order $2 n$ (or infinite order) may be found in [2] and [3]. Sweedler has a 4 dimensional (unpublished) example which differs from ours, discovered independently by the author, since the antipode of his example has order 2 if $k$ is a field of characteristic 2. To simplify the original description of our example, we follow the construction found on p. 89 of [3]. The author wishes to thank Dr. Robert G. Heyneman for his helpful suggestions during the writing of this paper.

1. Preliminary definitions and results. We define Hopf algebra and give basic properties of the antipode to facilitate description of the rank 4 example. Throughout this paper the definitions we use are those found in [1] and [3], except that we do not require $k$ to be a field. For the unproved assertions, the reader is referred to [1] or [3].

Let $k$ be a commutative ring. A $k$-bialgebra $(H, p, \Delta, \eta, \epsilon)$ is a $k$ module $H$ such that $(H, p, \eta)$ is a $k$-algebra and $(H, \Delta, \epsilon)$ is a $k$ coalgebra which are compatible in the sense that $\Delta$ and $\epsilon$ are algebra maps. We will frequently denote the bialgebra $(H, p, \Delta, \eta, \epsilon)$ by $H$.

If $H$ is a $k$-module, let $T: H \otimes H \rightarrow H \otimes H$ denote the "twist" map defined by $T(h \otimes k)=k \otimes h$. If $(H, p, \Delta, \eta, \epsilon)$ is a bialgebra, let $H^{\text {op }}$ denote the bialgebra $(H, p \circ T, \Delta, \eta, \epsilon)$ and $H^{\mathrm{cop}}$ denote the bialgebra $(H, p, T \circ \Delta, \eta, \epsilon)$. For a bialgebra $H$, we make End $H=\operatorname{Hom}_{k}(H, H)$ into an algebra, called the convolution algebra, where $\eta \circ \epsilon$ is the unit, and multiplication is defined by $f * g=p \circ f \otimes g \circ \Delta$, all $f, g \in$ End $H$.

Definition. Let $H$ be a bialgebra. If the identity operator $I$ $\in$ End $H$ has an inverse $s$ in the convolution algebra, then $H$ is called a Hopf algebra. The map $s$ is called the antipode of the Hopf algebra $H$.

Received by the editors August 27, 1970.

AMS 1970 subject classifications. Primary 17A24; Secondary 17A24.

Key words and phrases. Hopf algebra, antipode of Hopf algebra, free algebra, order of antipode. 
The antipode $s$ of a Hopf algebra $H$ is an algebra and coalgebra "antimorphism" meaning

1.1. $p \circ(s \otimes s) \circ T=s \circ p: H \otimes H \rightarrow H$, and $s \circ \eta: k \rightarrow H$;

1.2. $T \circ(s \otimes s) \circ \Delta=\Delta \circ s: H \rightarrow H \otimes H$, and $\epsilon \circ s=\epsilon: H \rightarrow k$.

By definition of the antipode and by 1.1 and 1.2 :

1.3. If $H$ is a Hopf algebra with antipode $s$, then $\left(H^{\text {op }}\right)^{\text {cop }}$ is a Hopf algebra with antipode $s$, and $H \rightarrow\left(H^{\circ p}\right)^{\text {cop }}$ is a map of Hopf algebras.

The following result (unpublished) due to Heyneman will be used in the next section.

1.4. Proposition. Let $H$ be a Hopf algebra with antipode s, and let $s^{\prime} \in$ End H. Then T.F.A.E.

(1) $s \circ s^{\prime}=s^{\prime} \circ s=I$.

(2) $H^{\mathrm{op}}$ is a Hopf algebra with antipode $s^{\prime}$.

(3) $H^{\text {cop }}$ is a Hopf algebra with antipode $s^{\prime}$.

Proof. $1 \Rightarrow 2$. Assume that $s \circ s^{\prime}=s^{\prime} \circ s=I$. Then

$$
\begin{aligned}
s \circ\left(p \circ T \circ\left(s^{\prime} \otimes I\right) \circ \Delta\right) & =p \circ\left(s \circ s^{\prime} \otimes s\right) \circ \Delta \\
& =p \circ(I \otimes s) \circ \Delta=\eta \circ \epsilon=s \circ(\eta \circ \epsilon)
\end{aligned}
$$

by 1.1. Since $s$ is injective, $(p \circ T) \circ\left(s^{\prime} \otimes I\right) \circ \Delta=\eta \circ \epsilon$. Likewise, $(p \circ T) \circ\left(I \otimes s^{\prime}\right) \circ \Delta=\eta \circ \epsilon$. Therefore, $H^{\circ p}$ is a Hopf algebra with antipode $s^{\prime}$.

$2 \Rightarrow 1$. Assume that $H^{\text {op }}$ is a Hopf algebra with antipode $s^{\prime}$. Then

$$
\begin{aligned}
p \circ\left(s \circ s^{\prime} \otimes s\right) \circ \Delta & =p \circ\left(s \otimes s \circ s^{\prime} \otimes I\right) \circ \Delta \\
& =s \circ\left(p \circ T \circ\left(s^{\prime} \otimes I\right)\right) \circ \Delta=s \circ(\eta \circ \epsilon)=\eta \circ \epsilon .
\end{aligned}
$$

Therefore, $p \circ\left(s \circ s^{\prime} \otimes s\right) \circ \Delta=\eta \circ \epsilon$ which implies that $s \circ s^{\prime}=I$. Likewise, $s^{\prime} \circ s=I$.

$2 \Leftrightarrow 3$. This follows by 1.3 . The proof is complete.

Let $H$ be a Hopf algebra with antipode $s$. Then $s$ is said to have order $n$ if $n$ is the least positive integer such that $s^{n}=I$.

If $(H, p, \Delta, \eta, \epsilon)$ is a bialgebra (Hopf algebra with antipode $s$ ) and $H$ is a free $k$-module of finite rank, then $\left(H^{*}, \Delta^{*}, p^{*}, \epsilon^{*}, \eta^{*}\right)$ is a bialgebra (Hopf algebra with antipode $s^{*}$ ).

2. The construction and basic properties of the example. Let $k\{Z, A\}$ be the free $k$-algebra on the symbols $Z$ and $A$. Then $\mathcal{H C}$ $=k\{Z, A\}$ has a unique bialgebra structure determined by

$$
\begin{aligned}
\Delta Z & =Z \otimes Z, & \epsilon(Z) & =1, \\
\Delta A & =A \otimes Z+1 \otimes A, & \epsilon(A) & =0 .
\end{aligned}
$$


Let $I$ be the ideal of $\mathcal{H}$ defined by $I=\left(Z^{2}-1, A^{2}-A\right.$, $Z A-(Z-(A Z+1)))$ and let $\mathfrak{H} \stackrel{\pi}{\longrightarrow} \mathfrak{H} / I$ be the projection. Then by direct computation one sees that $\pi \otimes \pi \circ \Delta(\alpha)=0$ and $\epsilon(\alpha)=0$ for each of the generators $\alpha$ of $I$. Therefore, $I$ is also a coideal, and $H=\mathfrak{H C} / I$ has an induced bialgebra structure. $H$ is a free $k$-module with basis (denoting cosets by small case letters) $1, z, a, b=a z$. In addition, $H$ has an antipode of order 4 determined by

$$
\begin{array}{ll}
s(1)=1, & s(a)=-b, \\
s(z)=z, & s(b)=a+z-1 .
\end{array}
$$

\begin{tabular}{|c|c|c|c|c|}
\hline & 1 & $a$ & $b$ & $z$ \\
\hline 1 & 1 & $a$ & $b$ & $z$ \\
\hline$a$ & $a$ & $a$ & $b$ & $b$ \\
\hline$b$ & $b$ & $-a$ & $-b$ & $a$ \\
\hline$z$ & $z$ & $z-(b+1)$ & $1-(a+z)$ & 1 \\
\hline
\end{tabular}

The multiplication of $H$ is determined by the table below.

Similar examples of Hopf algebras of finite rank with antipode of order 4 can be constructed by taking the quotient of $\mathcal{H C}$ by the bi-ideal (ideal and coideal) $I=\left(Z^{2 n}-1, A^{2}-A, Z A-\left(Z-\left(A Z+Z^{2}\right)\right)\right)$. The reader should compare this construction with that found on p. 89 of $[3]$.

We conclude this section with remarks on the structure of the rank 4 Hopf algebra $H$ with antipode $s$.

2.1. $H^{\text {cop }}\left(H^{\circ p}\right)$ is a Hopf algebra, and $H \simeq H^{\text {cop }}\left(H \simeq H^{\text {op }}\right)$ as Hopf algebras.

Proof of 2.1. Since $s$ is bijective, $H^{\mathrm{cop}}\left(H^{\mathrm{op}}\right)$ is a Hopf algebra with antipode $s^{\prime}$ determined by $s \circ s^{\prime}=s^{\prime} \circ s=I$ by 1.4. The $k$-linear bijection $H \stackrel{\sigma}{\rightarrow} H^{\text {oop }}$ determined by

$$
\begin{array}{ll}
\sigma(1),=1, & \sigma(a)=-b, \\
\sigma(z)=z, & \sigma(b)=-a
\end{array}
$$


is easily seen to be a map of bialgebras (hence Hopf algebras). Therefore, the composition $H \stackrel{\sigma}{\rightarrow} H^{\text {cop }} \stackrel{s^{\prime}}{\rightarrow} H^{\text {op }}$ is an isomorphism of Hopf algebras by 1.3 .

2.2. $H^{*}$ is a Hopf algebra, and $H \simeq H^{*}$ as Hopf algebras.

Proof of 2.2. $H^{*}$ is a Hopf algebra since $H$ is free and of finite rank. Let $\overline{1}, \bar{z}, \bar{a}, \bar{b}$ be the dual basis in $H^{*}$. Then the $k$-linear bijection $H \stackrel{\omega}{\rightarrow} H^{*}$ determined by

$$
\begin{array}{ll}
\omega(1)=\epsilon, & \omega(a)=\bar{z}, \\
\omega(z)=\bar{I}+\bar{a}-(\bar{b}+\bar{z}), & \omega(b)=-(\bar{b}+\bar{z})
\end{array}
$$

is an isomorphism of bialgebras (hence Hopf algebras). To prove this note that $\omega=\omega^{*}: H=H^{* *} \rightarrow H^{*}$. Therefore, it suffices to show that $\omega$ is an algebra map (or a coalgebra map).

2.3. $H$ is not semisimple. In fact $x=a+z-(b+1)$ is a left integral and $y=-(a+b)$ is a right integral for $H$.

Proof of 2.3. It is straightforward to verify that $h x=\epsilon(h) x$ and $y h=\epsilon(h) y$ for all $h \in H$. If $J$ is the ideal of $H$ generated by $x$ and $y$, then $J^{2}=(0)$. Therefore, $H$ is not semisimple.

\section{REFERENCES}

1. R. Heyneman and M. E. Sweedler, Affine Hopf algebras. I, J. Algebra 13 (1969), 192-241. MR 39 \#6876.

2. R. G. Larson, The order of the antipode of a Hopf algebra, Proc. Amer. Math. Soc. 21 (1969), 167-170. MR 39 \#1524.

3. M. E. Sweedler, Hopf algebras, Math. Lecture Note Series, Benjamin, New York, 1969. MR 40 \#5705.

University of North Carolina, Chapel Hill, North Carolina 27514 